\title{
Evaluating TORA, GRP and OLSR under Voice-IP Telephony Traffic Using OPNET
}

\author{
Gurjeet Singh $^{1}$, Gurbhej Singh ${ }^{2}$ \\ Assistant Professor, Department of Computer Science \& Engineering, \\ Shaheed Bhagat Singh State Technical Campus,(SBSSTC) (Formerly, SBS College of Engineering \& Technology)
}

Ferozepur (Punjab), India ${ }^{1,2}$

\begin{abstract}
MANET is a self-organizing and self-configuring network in which mobile nodes communicate with each other with the help of wireless connection. In A mobile ad-hoc network, links between nodes change very quickly because every mobile node in network has a full freedom to move in any direction. There is no need to define the path between any nodes. There are basically three types of protocols used for the communication between the nodes Reactive, Proactive and Hybrid. In this paper routing protocols GRP, OLSR and TORA are compared in Ad-hoc network on the basis of parameters delay, throughput and network delay. In this paper OPNET Modeler 14.5 is used for simulation.
\end{abstract}

\section{Keywords: MANET,TORA,GRP, OLSR, OPNET,Voice IP Telephony}

\section{INTRODUCTION}

A mobile ad hoc network (MANET) is self configuring network because all nodes of network communicate with each other without any permanent routing topology. Any node of MANET can leave the network without any permission. In this network all nodes interact with each other by using wireless connection. MANET consists of mobile hosts like tablet, laptop and mobile etc by using radio interface. All nodes of network are also act as router that has capability to receive and transmit data packet in right direction .Topology in MANET is of dynamic nature means route between source and destination nodes keep on changing. Main challenges of MANET are to maintain network and routing between nodes every time, Which is possible only when node have information about network and neighbour nodes.

Characteristics of MANET

- $\quad$ A MANET is self organising network that mean there is no need to take permission for entering new nodes or removing existing from network.

- $\quad$ MANET is energy constrained network.

- This is a bandwidth constrained network that results congestion problems.

- In adhoc network no central mechanism is required for managing network because this is an infrastructure-less network.

- $\quad$ Frequently changing routes in MANET.

- There is high bit error rate in adhoc network

\section{TYPES OF ROUTING}

In adhoc network there are three basic types of routing protocols reactive, proactive[15] and hybrid. Reactive routing protocol selects route according to the requirement. When there is a packet to transmit, protocol selects a suitable route from source to destination nodes and this route remain unchanged before reaching the destination node. AODV, DSR, TORA and ABR are examples of reactive protocol. Proactive routing protocol is based on the formula that routing protocol makes decision to get router, when there is need to transmit packet. In proactive protocol routes of packet changes very quickly before reaches to destination due to change in topology. DSDV, WRP, OLSR and CGSR protocols are example of proactive routing. Third type is hybrid protocol that work on combined features of reactive and proactive routing type protocols to enhance scalability for large network and higher node density. On certain conditions hybrid protocol combines features of reactive and proactive routing protocols. ZRP and CBRP are examples of hybrid type.

There are two types of routings static and dynamic routing in Mobile Adhoc routing

1. Static routing: Static is permanent routing technique. This type of routing is manually done by administrator to transmit and forward packets in adhoc network. In this routing, routing table is not required. There is no need to change entrees of routing table by routers itself.

2. Dynamic routing: This type of routing is done by router itself. Routers can route traffic in network on proper path with the help of routing table. Dynamic routing permits to router to get knowledge about network and to add required things in their routing tables. MANET is not permanent network it keeps on changing with this change network, routing table also change their contents according to need. Routers can add some new routes and removes old routes. This routing has capability to overcome traffic overload due to flexibility that dynamic routing have had. Dynamic routing is better than static routing it uses different paths to forward packets.

\section{III.Routing Protocols in Manets}

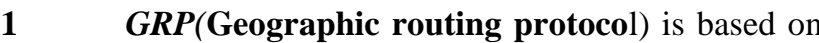
greedy routing and face routing principles[1].GRP is proactive routing protocol which is one of the position 
based protocols. GRP will mark the position of node and quadrants will optimize the flooding.Flooding position updates on distance the node moved and neighborhood crossings. Position of nodes and their neighbours is identified by exchanging hello protocol. A Node can return its packet to the last node when it can't keep on sending the packet to the next node by means of route locking at the same time.[2]The process of obtaining the location information is difficult or expensive in TORA for example GPS, and they don't work indoors. The methods to estimate locations are computation intensive, and with not good accuracy [3].

2. TORA (Temporally Order Routing Protocol) which is based on the concept of link reversal which is highly adaptive, loop-free, distributed routing algorithm[8,9].TORA is proposed to work in mobile networking environment which is highly dynamic.The localization of control messages to a very small set of nodes near the occurrence of a topological change is the main concept in design of TORA. To acheive above said objectives nodes need to maintain routing information about adjacent (one-hop) nodes[5].

The protocol performs three basic functions:

(a) Route creation, (b) Route maintenance, and (c) Route erasure.

Creating a route from a source node to destination node requires establishing a sequence of directed links from source to destination. This is accomplished using a query/reply process that builds a directed acyclic graph (DAG) rooted [4] at the destination. Reestablishing routes to destination in response to topological changes maintains the route. Query (QRY), update (UPD), clear (CLR) packets are used for creating routes, creating and maintaining routes, erasing routes respectively[6]. TORA possesses the following attributes[7]:

- Loop-free routes

- $\quad$ Provide minimal routing functionality

- Minimize algorithm reaction

- $\quad$ Multipath routing

2. Optimized Link State Routing (OLSR) protocol -OSLR is a proactive routing protocol used in adhoc networks. It is a table-driven protocol as it maintains and updates its routing table very quickly. OLSR exchanges the topology information always with other nodes. Few nodes are selected as MPRs (Multi point relays). MPRs are responsible for transmission of broadcast messages during flooding and generating link state information. MPRs technique used in OLSR protocol will reduce the message overhead and even minimize the number of control messages flooded in the network. Nodes maintain the information of neighbours and MPR's, by sending and receiving HELLO messages from its neighbours. Node A transmits the HELLO message to node $\mathrm{B}$ and then the message received by node $\mathrm{B}$ from node A can be called asymmetric link.

If this HELLO message is retransmitted by the node B to node $\mathrm{A}$ then the resulting link even called as asymmetric link. Finally the resulted bidirectional link is known as a symmetric link. Symmetric link formation will help the nodes to choose MPRs. MPRs will send the topology control (TC) messages containing the information about link status and MRP node information [10].

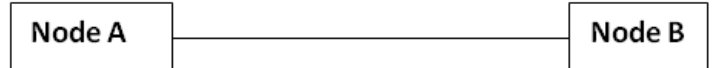

Hello Message -Asymmetric

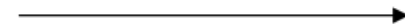

Hello Message -Symmetric

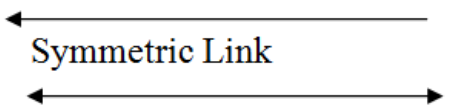

Fig.3.1 OLSR Symmetric Link Formation[11]

\section{IV.SIMULATION SETUP}

We have done analysis of GRP ,TORA and OLSR. The network area of $10 \mathrm{Km}^{*} 10 \mathrm{Km}$ is selected. The mobile nodes are deployed in network area. The 50 numbers of mobile nodes are taken with different protocols. The time for simulation is taken as 600 seconds and traffic used to simulation is voice IP Telephony. Voice IP Telephony application and profile is configured for this paper. The network is wireless LAN for mobile nodes having a data rate of $11 \mathrm{Mbps}$ and random waypoint model used for this simulation. DES's (global discrete event statistics) are collected on both protocols and wireless LAN. The mobility model used in this simulation is simple and it shows good mobility behaviour. Figure 4.1 shows simulation setup for 50 mobile nodes. The important parameters used to evaluate performance of all these three protocols are delay, network load and throughput.

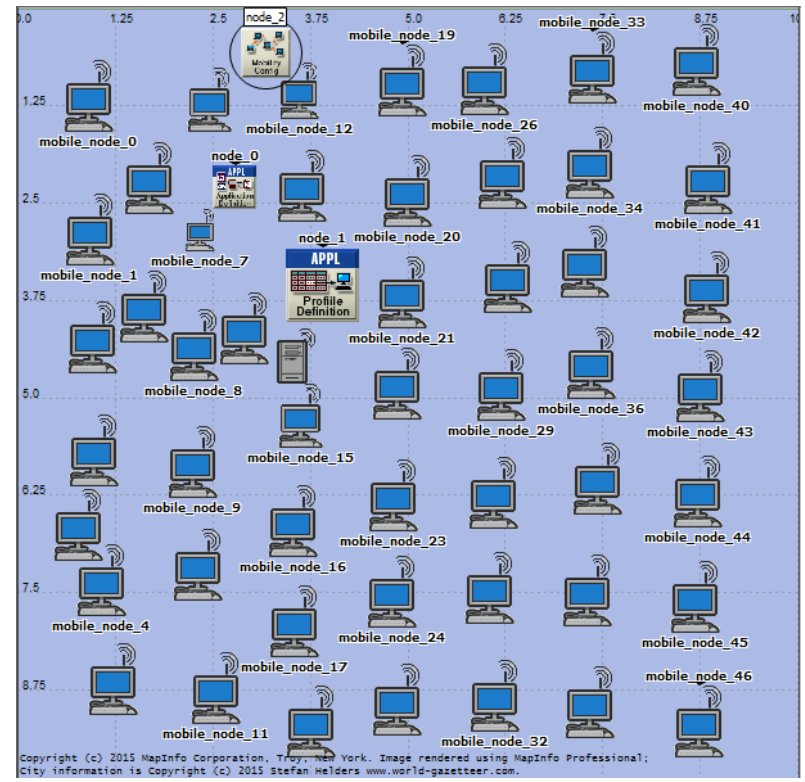

Fig. 4.1 Simulating environment for 50 Nodes 
International Journal of Advanced Research in Computer and Communication Engineering

Vol. 4, Issue 5, May 2015

Table 1 Simulation Parameter

\begin{tabular}{|c|c|}
\hline Routing protocol & GRP,TORA,OLSR \\
\hline MAC layer & 802.11 (DCF) \\
\hline MANET size & $10 \mathrm{Km} \mathrm{X} \mathrm{10} \mathrm{Km}$ \\
\hline Mobile nodes & 50 \\
\hline Mobility models & Random waypoint mobility \\
\hline Traffic type & Voice (IP Telephony) \\
\hline Simulation time & 600 Seconds \\
\hline $\begin{array}{c}\text { PHY standard } \\
\text { used }\end{array}$ & $802.11 \mathrm{~b}$ \\
\hline Data Rate & $11 \mathrm{Mbps}$ \\
\hline
\end{tabular}

\section{Software Environment}

In this paper Optimized Network Engineering Tool (OPNET v14.5) software is used for simulations. OPNET is a network simulator. OPNET provide us multiple solutions handling networks and applications e.g. operation on network, planning, research and development, performance management. Performance of network protocols and related technology can be compared by OPNET 14.5 .

OPNET simulator can provide solutions for the academic research, like improvement in wireless network technologies such as UMTS (Universal Mobile Telecommunications System) and Wi-Fi.OPNET is used to compare protocols under different traffics. IN MANET this simulator is used for wireless communication.

The OPNET have four main steps in working.

1. Network Modelling (creating network).

2. Choose of statistics selection of statistics.

3. Simulating the network.

4. View and analyze results related to network.

\section{Mobility Model}

In research community random waypoint model is most commonly used model.A node randomly chooses a destination and moves towards it with a velocity chosen randomly from a uniform distribution $\left[0, \mathrm{~V}_{\max }\right]$ at every instant where $\mathrm{V}_{\max }$ is the maximum allowable velocity for every mobile node. The node after reaching the destination stops for a duration defined by the 'pause time' parameter.After this duration, it again chooses a random destination and repeats the whole process until the simulation ends.

\section{Performance Parameters}

The Voice IP Telephony traffic is considered here for the simulations since it is applicable in internet applications.

\subsection{Delay}

The Time of generation of a packet by the source up to the destination reception is end to end delay. So delay is time taken by packet to go across the network. Delay is expressed in seconds. Processing delay (PD), queuing delay (QD), transmission delay (TD) and propagation delay (PD)are called packet end to end delay in network. Latency time and delay has same meaning.

\subsection{Network Load}

Network load represents the total load in bit per second submitted to wireless LAN layers by all higher layers in all WLAN nodes of the network is called Network Load. [13]

\subsection{Throughput}

Throughput is ratio of the total data reaches a receiver from the sender. The time it takes by the receiver to receive the last message is called as throughput [12]

Topology changes in the network, unreliable communication between nodes, limited bandwidth available and limited energy are the factors that affects the throughput.Throughput is expressed as bytes or bits per sec (byte/sec or bit/sec). Throughput can be defined by the following formula [14]:

Throughput $=$ (number of delivered packet $*$ packet size)/total duration of simulation

\section{RESUltS AND DisCUSSION}

The protocols (GRP, TORA and OLSR) are evaluated under varies conditions to check their performance in the network field. Result part of this paper includes checking performance of given protocols with the help of parameters like delay, total network load and throughput and some other metric related to this field. Performances of given protocols are represented with the help of OPNET by drawing graphs.

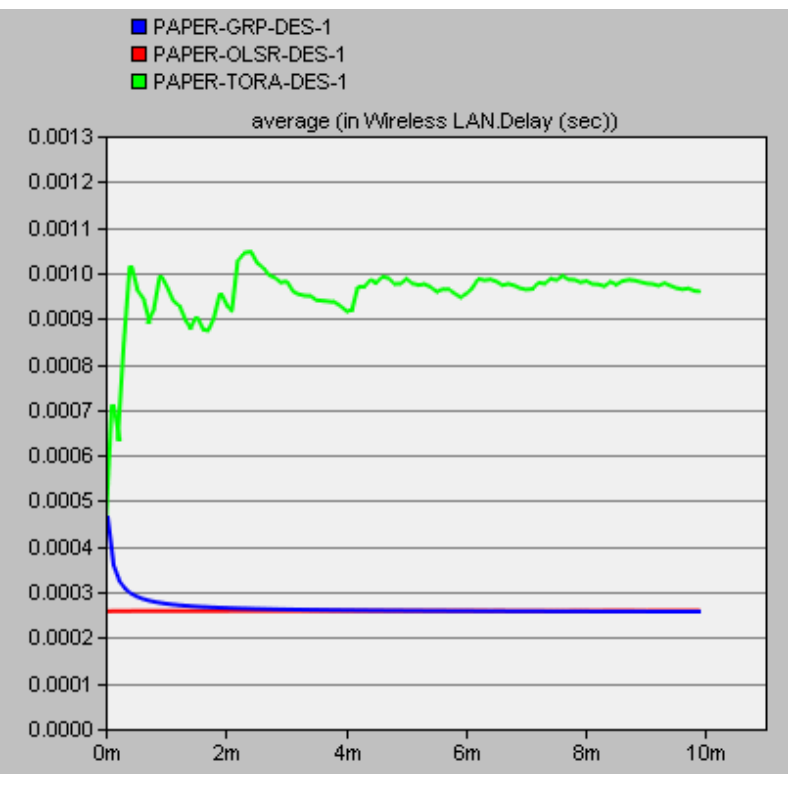

Fig 5.1 Measurement of Delay in 50 nodes for GRP,OLSR and TORA

Figure 5.1 represents delay (seconds) for GRP, TORA and OLSR when there are 50 mobile nodes in network of $10 \mathrm{~km} * 10 \mathrm{~km}$. Given graph shows that TORA protocol has 
maximum delay and GRP has minimum delay under given conditions. The graph shows that in TORA protocol delay increases with time and after some time becomes constant. Initially TORA has less delay and then rise rapidly But GRP protocol behaves completely in different way. The graph shows that in GRP delay reduces after some time and become constant. Delay in OLSR protocol remains constant for network lifetime. In Fig 5.1 multicolour lines represents delay for protocols GRP, TORA and OLSR respectively.

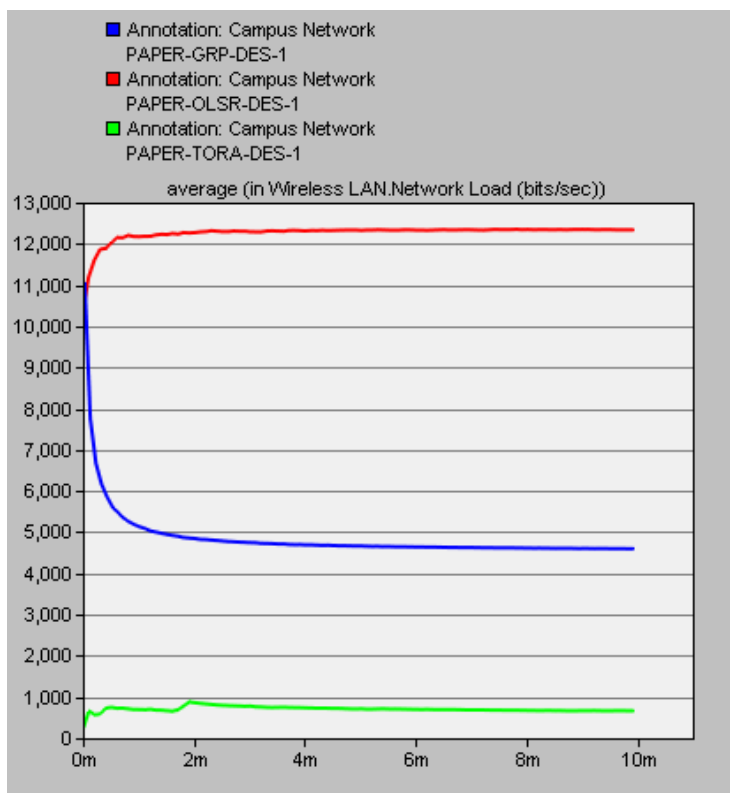

Fig 5.2 Measurement of Network Load in 50 nodes for GRP,OLSR and TORA

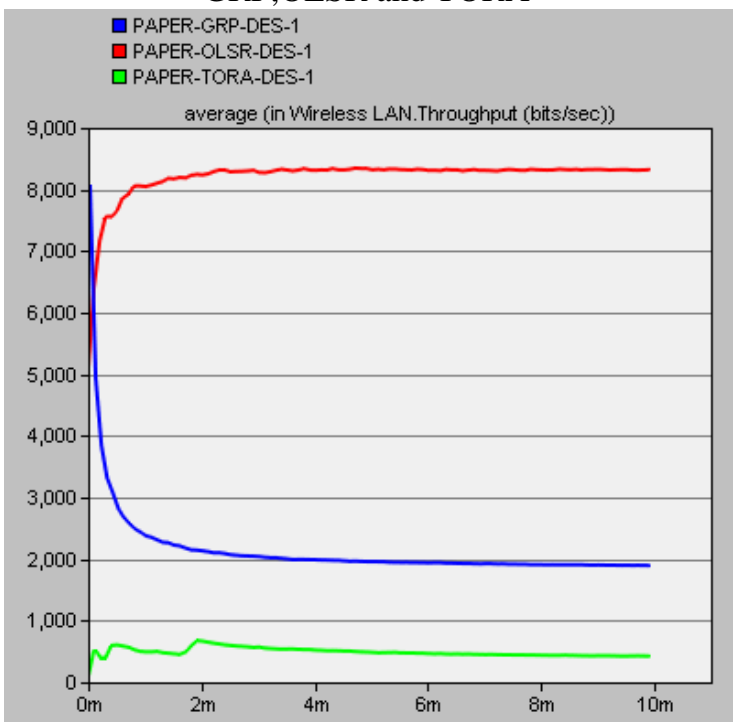

Fig 5.3 Measurement of Throughput in 50 nodes for GRP,OLSR and TORA

Figure 5.2 network load (bit/seconds) for GRP, TORA and OLSR when there are 50 mobile nodes in network of $10 \mathrm{~km} * 10 \mathrm{~km}$. Given graph shows that TORA protocol has very less network load and OLSR has maximum network load under given conditions. The graph shows that in TORA protocol has very less variation in network load.
But in GRP protocol network load is less than OLSR and more than TORA. The graph shows that GRP in network load reduces after some time and become constant. Network load in OLSR protocol is maximum and it increase with time.

Figure 5.3 Throughput (bit/seconds) for GRP, TORA and OLSR when there are 50 mobile nodes in network of $10 \mathrm{~km} * 10 \mathrm{~km}$. Given graph shows that TORA protocol has very less Throughput and OLSR has maximum Throughput under given conditions. The graph shows that in TORA protocol has very less variation in network load. But in GRP protocol Throughput is less than OLSR and more than TORA. The graph shows that GRP in Throughput reduces after some time and become constant. Throughput in OLSR protocol is maximum and it increase with time.

\section{CONCLUSION}

The OPNET simulater 14.5 is used for analysing comparative study of GRP, TORA and OLSR. It is observed from results and conclusion part that DSR protocol has high throughput and very less access delay and average network load so this best protocol in this condion. TORA protocol has very poor throughput as compared to both protocols DSR and OLSR but on other side TORA shows minimum network delay. The Qos can be different for these three protocols if it observed under different parameters or simulation conditions.But for particular conditions TORA provides best overall performance.

\section{REFERENCES}

[1] Anand, G.C.S.; Vaidya, R.R.; Velmurugan, T., "Performance Analysis of VoIP Traffic using various Protocols and Throughput enhancement in WLANs," Computer, Communication and Electrical Technology (ICCCET), 2011 International Conference on , vol., no., pp.176,180, 18-19 March 2011 doi: 10.1109/ICCCET.2011.5762463

[2] Li Zhiyuan, "Geographic Routing Protocol and Simulation," Computer Science and Engineering, 2009. WCSE '09. Second International Workshop on , vol.2, no., pp.404,407, 28-30 Oct. doi: 10.1109/WCSE.2009.840

[3] Stephan Ruhrup, "Theory and practice of Geographic Routing", December 2009.

[4] Park VD, "A highly adaptive distributed routing algorithm for mobile wireless networks", Proceedings of IEEE INFOCOM 1997, Volume 3: 1405-1413

[5] Sharma, S.S.; Hijam, R.; Barun, K.; Rajkumari, R., "Issues of MANET routing protocols on $4 \mathrm{G}$ network," Computing, Communications and Networking Technologies (ICCCNT),2013 Fourth International Conference on , vol., no., pp.1,8, 4-6 July 2013 doi: 10.1109/ICCCNT.2013.6726697

[6] Dadral, P.; Vohra, R.; Sawhney, R.S., "Metrics improvement of MANET using reactive protocols approach," Parallel Distributed and Grid Computing (PDGC), 2012 2nd IEEE International Conference on, vol., no., pp.73,78, 6-8 Dec. 2012 doi: 10.1109/PDGC.2012.6449794

17] Rajput, M.; Khatri, P.; Shastri, A.; Solanki, K., "Comparison of Adhoc reactive routing protocols using OPNET modeler," Computer Information Systems and Industrial Management Applications (CISIM), 2010 International Conference on , vol., no., pp.530,534, 8-10 doi: 10.1109/CISIM.2010.5643454

[8] Al-Ghadanfary, K.A.; Al-Somaidai, M.B., "Simulation of some Routing Protocols in a client wireless mesh network for multimedia 
applications," Electrical, Communication, Computer, Power, and Control Engineering (ICECCPCE), 2013 International Conference on, vol., no., pp.107,112, 17-18 Dec. 2013 doi: 10.1109/ICECCPCE.2013.6998743

[9] Park, V. D. and Corson, M. S. "A Performance Comparison of the Temporally Ordered Routing Algorithm and Ideal Link-State Routing", Proceedings of IEEE Symposium on Computers and Communication'98, pp. 592-598, Athens, Greece, June 1998

[10] T. Clausen, P. Jacquet "Optimized Link State Routing Protocol (OLSR)" NWG, 2003. [Online]. Available: http://www.ietf.org/rfc/rfc3626.txt [Accessed: Mar 28,2010]

[11] Y.Ravi Kumar ,S.K.Chittamurer,"A case study on routing protocols performance over TCP and HTTP",Master Thesis No. MEE 10:49 June 2010

[12] Uyen Trang Nguyen and Xing Xiong, "Rate-adaptive Multicast in Mobile Ad-hoc Networks", Department of Computer Science and Engineering York University Toronto, Canada M3J 1P3.

13] Suhaimi Bin Abd Latif, M.A. Rashid, F.Alam, "Profiling Delay and Throughput Characteristics of Interactive Multimedia Traffic over WLANs using OPNET", School of Engineering Institute of Technology \& Engineering, Massey University, Auckland, New Zealand

[14] Parulpreet Singh, Ekta Barkhodia, Gurleen Kaur Walia ,"Evaluation of various Traffic loads in MANET with DSR routing protocol through use of OPNET Simulator", International Journal of Distributed and Parallel Systems (IJDPS) Vol.3, No.3, May 2012 DOI : 10.5121/ijdps.330875,2012

[15] Gurbhej Singh,Gurjeet Singh," Remote login based traffic Performance Analysis of Reactive Routing Protocols of MANETs using OPNET", International Journal of Advanced Research in Computer and Communication Engineering Vol. 4, Issue 2, pp 438441 February 2015 